\title{
Origin of waves in surface-tension-driven convection
}

\author{
Angel Garcimartín, ${ }^{1, *}$ Nathalie Mukolobwiez, ${ }^{2}$ and François Daviaud ${ }^{2}$ \\ ${ }^{1}$ Departamento de Física, Facultad de Ciencias, Universidad de Navarra, E-31080 Pamplona, Spain \\ ${ }^{2}$ Service de Physique de l'Etat Condensé, CEA Saclay, F-91191 Gif-sur-Yvette, France
}

(Received 10 June 1996; revised manuscript received 7 March 1997)

\begin{abstract}
Waves appear in a liquid layer with a free surface if a sufficiently high horizontal temperature gradient is imposed. These waves have been compared to the hydrothermal waves predicted by a linear stability analysis of a parallel flow. However, depending on the experimental configurations, significant differences with theory are found. We show that there exists another kind of wave that cannot be explained by previous analysis. Our aim is to investigate which is the mechanism leading to this instability. Differential interferometry is used to obtain quantitative information on the temperature field. Experimental evidence is presented suggesting that these waves are the result of a boundary layer instability: the roll near the hot wall begins to oscillate, and the perturbations are dragged and amplified downflow. This mechanism could explain discrepancies between theory and some experimental observations. [S1063-651X(97)04108-1]

PACS number(s): 47.20.Dr, 47.20.Bp, 47.27.-i
\end{abstract}

\section{INTRODUCTION}

A fluid layer heated from one side (and cooled from the opposite) gets into motion, no matter how small the imposed temperature difference $\Delta T$ between the end walls is. Two unbalanced forces set up a global flow. The first one arises from the change of the surface forces with temperature, also called the Marangoni effect. The second one is gravity, acting upon the density variations with temperature. The two main experimental parameters, the temperature difference $\Delta T$ and the depth of the fluid layer $h$, can be expressed in terms of two nondimensional numbers: the Marangoni number Ma and the Rayleigh number Ra. We use the following definitions for $\mathrm{Ra}$ and Ma:

$$
\mathrm{Ra}=\frac{\alpha g \beta h^{4}}{\nu \kappa}, \quad \mathrm{Ma}=\frac{\frac{d \sigma}{d T} \beta h^{2}}{\mu \kappa},
$$

where $\alpha$ is the thermal expansion coefficient, $g$ is the gravity, $\beta$ is the imposed (horizontal) temperature gradient, $h$ is the layer depth, $\nu$ is the kinematic viscosity, $\kappa$ is the thermal diffusivity, $\sigma$ is the surface tension, and $\mu$ is the dynamic viscosity. The ratio $\mathrm{Ra} / \mathrm{Ma}$, sometimes called the dynamic Bond number, depends on $h^{2}$, and gives the relative importance of the gravity and surface tension forces. Thus for small $h$ the Marangoni effect dominates, while for large depths gravity effects overcome the surface tension forces. There is still another nondimensional number: the Prandtl number $\operatorname{Pr}$, defined as $\operatorname{Pr}=\nu / \kappa$, that accounts for the relative importance of thermal conduction and viscous dissipation. The geometry of the container is defined by two aspect ratios, namely, $\Gamma_{x}$ and $\Gamma_{y}$. The first one corresponds to the

\footnotetext{
*Present address: Laboratoire de Physique, Ecole Normale Supérieure de Lyon, 46 Allée d'Italie, F-69364 Lyon Cedex 07, France. Electronic address: agarcima@physique.ens-lyon.fr
}

distance between hot and cold walls divided by the height of the fluid layer; the second to the transversal dimension of the cell divided by the height.

In this work, we examine shallow layers, where surface tension effects are more relevant than gravity. The liquid used has a moderate Pr number, moderate meaning between 10 and 100 , so the velocity follows the temperature field. Considerable interest in this configuration comes from several industrial processes taking place in similar situations: the fabrication and purification of high-quality crystals, electron-beam vaporization of metals and laser welding, for instance. Experiments are being carried out to explore the possibility of manufacturing crystals in space, where gravity is negligible and only surface tension forces are relevant. The knowledge of the mechanisms leading to instabilities as the control parameter $\Delta T$ increases should help to improve these industrial procedures.

An analytical expression for this basic flow can be found, and experiments [1] showed that this description is accurate. Except near the end walls, the flow is horizontal, from hot to cold near the surface, and in the other sense near the bottom. It is therefore two dimensional in the core. Starting from this situation, several studies-both theoretical and experimental-have tried to describe the flow structure as $\Delta T$ is increased. As soon as the basic flow becomes unstable, different kinds of phenomena have been observed in several experiments, some of which differ from the theoretical analyses.

Smith and Davis [2] performed a stability analysis for an infinite fluid layer with a free surface, where a constant horizontal temperature gradient was imposed. They did not take into account neither gravity nor heat exchange to the atmosphere. They found the most dangerous modes for a range of Pr numbers and provided the corresponding instability thresholds. For a range of small to moderate Pr numbers, they found that the instability was oscillatory, coining the term "hydrothermal waves."

Some experiments, carried out independently by several groups $[1,3-5]$ indeed showed the existence of waves. But some intriguing features were also found that could not be 
explained within the theoretical results. Both Schwabe et al. [4] and Ezersky et al. [5] observed a different sequence of states as they increased the control parameter $\Delta T$ in a large $\Gamma_{x}$ configuration. In fact, before waves showed up, stationary corotative rolls were observed with their axes oriented perpendicularly to the temperature gradient. This new state had not been predicted. Villers and Platten [1] performed careful velocity measurements on a narrow channel $\left(\Gamma_{y} \approx 1\right)$ differentially heated at the ends, and they also found stationary corotative rolls that have been studied in new experiments with large $\Gamma_{x}$ [6]. While the basic flow was found to be correctly described by theory, these stationary corotative rolls are clearly a depart from it. Daviaud and Vince [3] used a cell with a large $\Gamma_{y}$ and small $\Gamma_{x}$. They found rolls for large depths. In this case, the rolls are counter-rotating and oriented in the same direction as the temperature gradient.

Other theoretical and numerical studies followed, starting from a different hypothesis. Gravity, for example, was taken into account $[7,8]$, and the Biot number-which gives the heat loss to the air-was assumed to depart from zero [9]. It was consistently found that instabilities were waves propagating with a given angle against the temperature gradient, or stationary counter-rotating rolls aligned with the temperature gradient, depending on the value of the parameters [9].

In the experiments it is observed that starting from rolls a further increase of $\Delta T$ leads to the appearance of waves, which were found and described experimentally by Daviaud and Vince [3], Schwabe et al. [4], and Ezersky et al. [5] in different geometries. Oscillations were also found by Villers and Platten [1], but the constraints of their experimental setup did not allow a detailed study. Some features of the waves-such as frequency-seemed to fit the theoretical descriptions, but there were, however, significant discrepancies. In some cases, waves were found to travel at an angle and/or direction different to the predicted $[10,4]$. In other cases [3], waves resembled more closely the theoretical studies [9], traveling at a certain angle against the temperature gradient.

In summary, theoretical analyses reproduce some features, but none can explain all the observed behaviors. Here we address the question of the physical mechanism lying at the origin of the waves, in an effort to elucidate these discrepancies. In order to do that, we have set up an experiment to observe the hot end of a fluid layer. We chose interferometry because it is a noninvasive yet sensitive method.

In Sec. II, experimental procedures are described. Results are presented in Sec. III. A brief discussion-where we venture a possible explanation of the phenomenon-is elaborated upon in Sec. IV. In Sec. V, some conclusions are provided.

\section{EXPERIMENTAL SETUP}

Three liquids have been used: silicone oils 47V5 and 47V0.65 from Rhône-Poulenc, and decane. Their Prandtl numbers are respectively 30,10 , and 15 at $20^{\circ} \mathrm{C}$. The phenomenology presented in this paper is basically the same for these three liquids. In the following, we will just give one relevant figure of any of the liquids. It should be understood that the physical parameters for which the same phenomenon is observed in the other liquids may change.

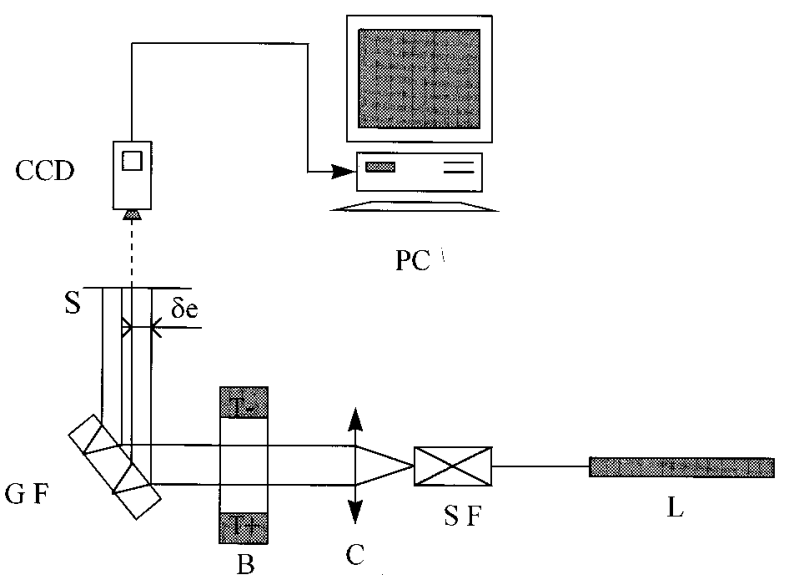

FIG. 1. Sketch of the experimental setup. $L$ : laser; SF: spatial filter; $C$ : collimating lens; $B$ : convective cell; GF: glass flat; $S$ : screen; CCD: camera; $\delta e$ : lateral shear; PC: computer.

The box used in this experiment is made of glass of good optical quality whose surfaces are polished to one wavelength or better. It is $10 \mathrm{~cm}$ long and $1 \mathrm{~cm}$ wide. The width of the cell cannot be increased significantly without producing so large a deflection in the laser beam that interferometry becomes impractical. Two metal prismatic pieces, whose section is $1 \times 1 \mathrm{~cm}^{2}$, are placed inside the box, at the two ends, to provide isothermal heating and cooling. Water is circulated through these pieces from thermal baths where temperature is fixed with a precision better than $0.05^{\circ} \mathrm{C}$. A thermocouple is attached to each metal piece near the bottom to register the temperature at the end walls. The air temperature is also monitored, although the heat exchange to the atmosphere cannot be controlled.

Various interferometry setups are commonplace. As typical temperature gradients have an order of magnitude of $1{ }^{\circ} \mathrm{C} / \mathrm{cm}$, an extremely sensitive apparatus would produce a lot of interference fringes for our system. Instead, a differential interferometry, in one of the variations of the Jamin interferometer [11], has been used. We provide a sketch of our setup in Fig. 1. A polarized He-Ne laser of $5 \mathrm{~mW}$ is filtered and collimated. The expanded beam is sent horizontally to the convective box, perpendicularly to the temperature gradient. After going through the liquid, the beam arrives at a glass flat polished to one wavelength, where the beam is reflected in both the first and second surfaces. These two reflections interfere. The interferogram is captured with a standard video camera and sent to a computer for acquisition and processing. A video recorder connected to the system allows one to register a movie of dynamic events.

In our setup, the two interfering beams have both passed through the liquid. In other interferometers, for instance in the Mach-Zender interferometer, the beam is split and one of the beams travels outside the object under study, thus providing an absolute reference when recombining with the beam that has gone through the object. This is not the case here. The information that can be obtained from our interferometer concerns the temperature difference between the zones crossed by the rays that interfere. If they are close enough, one can take the local temperature gradient instead of the temperature difference. If we call $k$ the interference 
order, then the difference of $k$ between two points of the interferogram reads [12]

$$
\delta(k)=l \frac{\delta e}{\lambda} \frac{d n}{d T} \delta\left(\frac{d T}{d x}\right) .
$$

In this formula, $l$ is the length of fluid crossed by the laser beam, i.e., the transversal dimension of the layer; $\delta e$ is the spacing between two interfering rays when entering the cell, sometimes called the lateral shear of the apparatus, and its value can be calculated from the thickness of the optical flat and its inclination; $\lambda$ is the light wavelength; $d n / d T$ is the variation of the refractive index with temperature; and $d T / d x$ is the local temperature gradient in the direction of the shear introduced by the interferometer. In our setup, $l$ is $1 \mathrm{~cm} ; \lambda$, the wavelength of the He-Ne laser, is $0.6348 \mu \mathrm{m}$, and the lateral shear $\delta e$ is $3 \mathrm{~mm}$ and has the same orientation than the temperature gradient (see Fig. 1). It follows that an interference fringe, whose $k$ is constant, is also a line where the local temperature gradient is constant. All the factors multiplying the temperature gradient can be calculated from the properties of the interferometer except $d n / d T$. We have obtained this value by measuring $n$ with an Abbe refractometer for the liquids we use. The values of $d n / d T$ obtained for 47V5 and 47V0.65 silicone oils and decane are, respectively, $-3.7 \times 10^{-4}{ }^{\circ} \mathrm{C}^{-1},-6.3 \times 10^{-4}{ }^{\circ} \mathrm{C}^{-1}$, and -4.9 $\times 10^{-4}{ }^{\circ} \mathrm{C}^{-1}$. The relative precision of the coefficient of $\delta(d T / d x)$ in Eq. (2) is about $10 \%$.

\section{RESULTS}

Our primary interest is to characterize the fluid layer destabilization. In particular, we want to study the waves which emerge above a temperature gradient threshold. We first turn to the basic flow, which is a well-characterized state, to test our experimental method. To understand the meaning of the interferogram, we need to obtain a relation between the temperature field and the interference order $k$. This is an inverse problem for which the general solution is not easy to find. But we can attempt to extract some quantitative information from the interferometry if we include previous knowledge, gathered by other means, on the temperature field. We indeed know that corotative rolls appear for given values of the control parameters; if we take a temperature field sinusoidally varying along both $x$ and $y$, and calculate the constant gradient lines, we find a pattern of concentric fringes. Things are not so simple in the real system, where the rolls are inclined, but every pair of these concentric patterns can be identified with a roll (Fig. 2).

Temperature measurements with thermocouples provide an independent test for the interferometry. However, we have estimated that thermocouple probes-even if they are very small-may yield a systematic error as high as $0.2^{\circ} \mathrm{C}$. Taking this remark into account, we present, in Fig. 3, the temperature profile along the cell obtained with a thermocouple at the depth where the amplitude of the rolls is larger. After subtracting the constant temperature gradient between the two walls, the roll at about $2.5 \mathrm{~cm}$ from the hot side is found to have an amplitude of about $0.3^{\circ} \mathrm{C}$.

The roll shown in Fig. 2 has been obtained under the same conditions, i.e., the same Ma and $\mathrm{Ra}$, and at the same place. a)

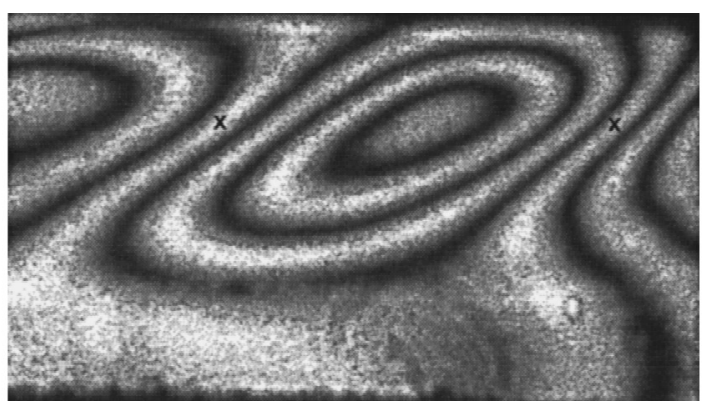

b)

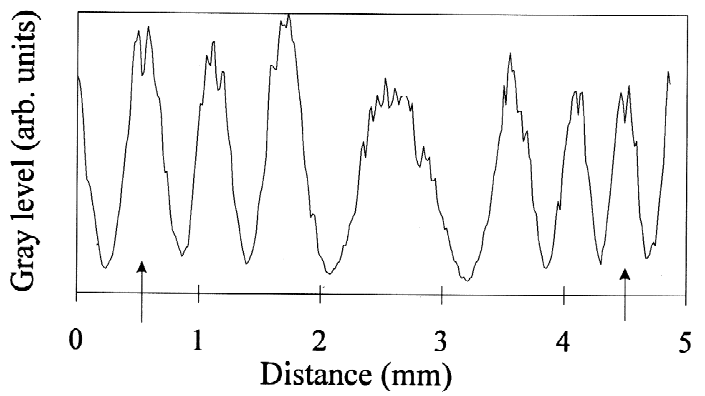

c)

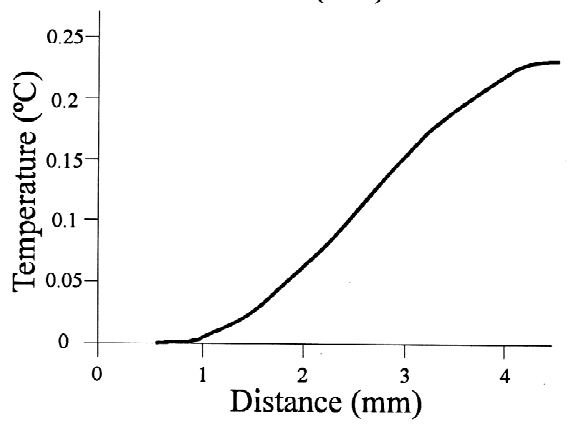

FIG. 2. (a) Interferogram obtained at $2.4 \mathrm{~cm}$ from the hot wall (left side). The dimensions of the zone displayed are $3 \mathrm{~mm}$ high (the whole liquid layer) by $7 \mathrm{~mm}$. Note that at the points marked with $X$ the temperature gradient is the same than in the background. (b) Light intensity along a segment passing through the two points marked with $X$ in (a). The two points marked with $X$ are indicated here with arrows. To find the amplitude of the roll, superimposed on a constant temperature gradient, the temperature gradient is taken to be zero at those points for the calculation. A temperature gradient can be assigned to each maximum and minimum of this line after Eq. (2). (c) The temperature profile resulting from the above-mentioned calculation (the origin of temperatures is arbitrary). One can see that a concentric set of fringes is equivalent to half a roll.

Assuming a constant background temperature gradient-note that a constant temperature gradient gives no fringes-we can calculate the roll amplitude. We proceed in the following way. We take the light intensity along a line going through approximately the middle of the roll [see Fig. 2(b)]. For every two consecutive maxima of light intensity in the image, $\delta(k)$ is equal to 1 . The temperature gradient for these points is given by Eq. (2). The gradient for every intensity minimum can also be obtained, but an interpolation for all the curves is not reasonable because of the nonlinearities in the detection system. Once a set of temperature gradients is obtained at different points along the line, a numerical integration is performed on their interpolation, and the temperature along that line is obtained: see Fig. 2(c). The amplitude of 


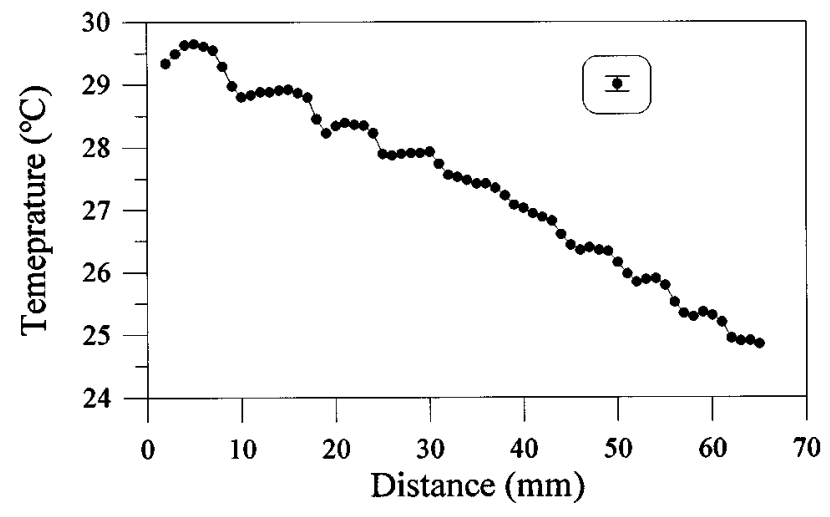

FIG. 3. Temperature profile along the cell, obtained with a thermocouple for the same conditions as Fig. 2. Rolls are seen over a constant background temperature gradient of about $0.7^{\circ} \mathrm{C} / \mathrm{cm}$. The amplitude of the roll located at about $2.4 \mathrm{~cm}$ from the hot wall is about $0.3^{\circ}$, which is consistent with the result shown in Fig. 2. The error bar is the standard deviation from the mean of a large number of consecutive measurements; the precision, however, might be much smaller due to the systematic perturbation introduced by the thermocouple.

the roll is about $0.25{ }^{\circ} \mathrm{C}$, which is in agreement with the thermocouple measurements. Assuming the temperature field will adopt some known configuration, one can therefore extract quantitative information from the interferometry.

Above a temperature gradient threshold, the flow becomes unstable, and the rolls begin to oscillate. Indeed, we observed waves propagating from the hot side to the cold side. So we tried to visualize the region just near the hot wall, imposing a temperature difference such as to produce waves in the fluid layer. A roll whose amplitude is much larger than that of the other rolls in the cell can be seen there. This roll begins to oscillate: above a certain temperature threshold, waves are released. They are detached from the roll near the surface (Fig. 4). The tip of the roll observed in Fig. 4 moves back and forth. Each time it retracts, it releases a "drop" of hot fluid near the surface; cold fluid is seen to rise from the bottom and to join the hot "drop." This can be seen in the interferogram as two concentric sets of fringes. The ensemble is dragged downflow, and constitutes one wave (in Fig. 5, one of these waves is shown at the middle of the cell). The picture we get from this scenario is quite similar to that observed by Dubois and Bergé in Rayleigh-Bénard convection [13]. They identified the oscillation mechanisms with boundary layer instabilities. In our case, these instabilities cause the release of a perturbation with a perturbation that is dragged by the flow.

The frequency of the waves has been described elsewhere $[4,10]$. It is interesting to check that the rotation period of the roll adjacent to the hot wall is consistent with the frequency already reported. We measured this period in two ways. First, aluminum particles were seeded and tracked (see Fig. 6). In the second method, a fine resistive wire was placed inside the roll and a short temperature pulse was released. With a thermocouple placed elsewhere in the roll, the time of travel can be measured. This latter method is obviously more perturbative than the former, but timing is much easier. With both methods a period of about $1 \mathrm{~s}$ is obtained in a situation

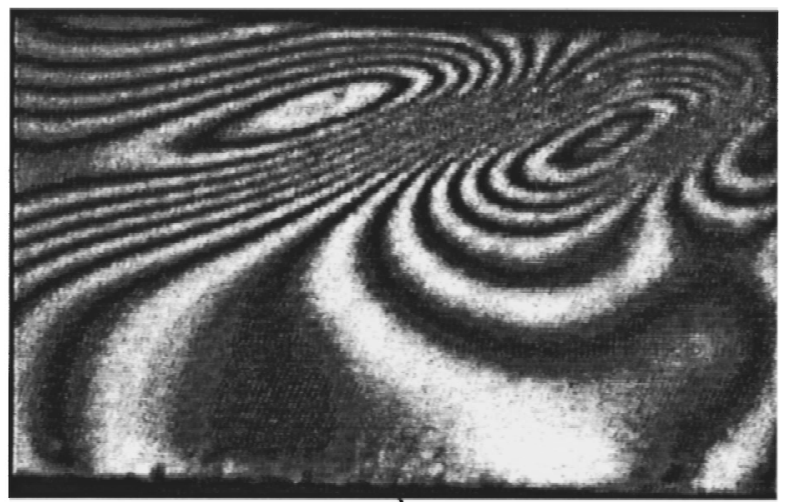

a)

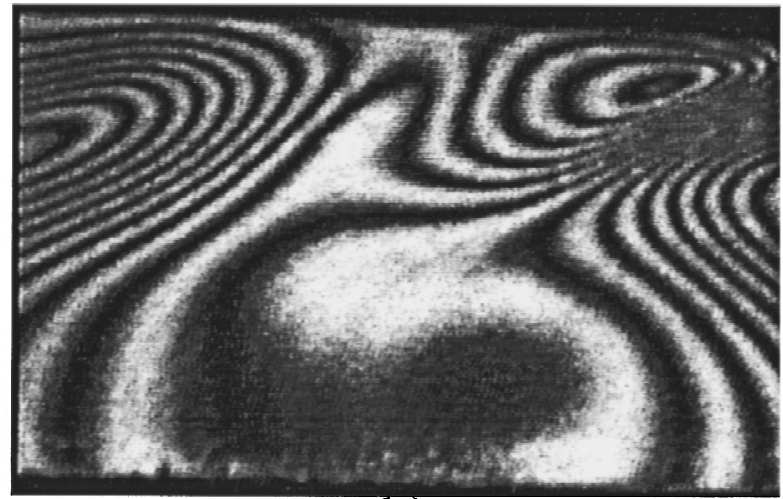

b)

FIG. 4. The tip of the firs roll is shown in these two shots at two stages of the oscillation. In (a) the tip (the protruding set of lines at the left upper half) has advanced and it is about to release a hot "drop." In (b) the hot "drop" has been released, and the tip of the roll is retracted. The concentric set of fringes that can be seen in (a) at the right half of the picture is the cold "drop" of fluid that has risen from the bottom and has joined the previous hot "drop." [The hot wall is at the left, and the photographs cover the same area as Fig. 2(a).]

where waves were measured to have a frequency of $1.2 \mathrm{~Hz}$.

It should be noted that in this roll the velocity is not at all constant. Near the hot wall the fluid accelerates, reaching a maximum speed near the surface. At that point the fluid turns abruptly and leaves the hot wall parallel to the surface. The return travel to the hot wall is much slower. Therefore the roll is not symmetric.

A measurement similar to that presented in Fig. 2 can be carried out for waves, which are in fact small traveling rolls. The temperature amplitude for the wave shown in Fig. 5 is given in Fig. 7. The value obtained is in agreement with measurements obtained from thermocouples (Fig. 8). It can be seen that the waves are much stronger near the surface, not only from thermocouple measurements, but from interferometry as well: fringes are more compressed in the upper part. Some amplification mechanism, maybe due to a surface instability, must be present, as shown in Ref. [10].

We complemented these observations with the shadowgraph method (Fig. 9). The cell is shown from the side, and an arrow marks the position where waves detach from the first roll. The first roll oscillates at a frequency of about $1 \mathrm{~Hz}$, and the waves are produced accordingly at this frequency. 


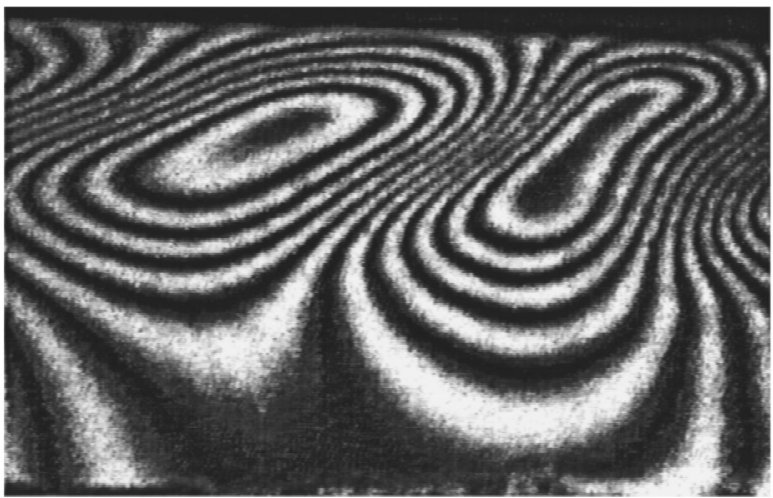

FIG. 5. A wave at about the middle of the cell [the dimensions of this picture are the same as in Fig. 2(a)]. The hot and cold "drops" have joined, forming a roll that moves toward the cold side (at the right). It can be seen that the amplitude of the wave is larger near the surface.

\section{DISCUSSION}

In this section we show that the waves we observed differ from the hydrothermal waves predicted by theory. We then provide some qualitative arguments in favor of another mechanism that might explain the experimental results presented in Sec. III.

There exist notable differences between these waves and hydrothermal waves. First, there is the parameter space where they show up. Hydrothermal waves appear only at small depths of fluid and for smaller temperature differences [3] than the waves we report here. Second, the direction of propagation is different. Hydrothermal waves go from the cold to the hot side, with the wave vector oriented at a given angle to the temperature gradient [2,7-9]. In our case, the waves travel from the hot to the cold end, with the wave vector perpendicular to the temperature gradient. We propose that the physical origin of both waves is different. Additional work on the subject is in progress [14,15]. Waves traveling in the same direction as the temperature gradient seem to be nothing but the result of the oscillation of the first roll that the flow carries down and amplifies. The observations we carried out strongly suggest that this could be the underlying process that gives rise to the waves traveling from the hot to the cold wall.

As we have stated, the first roll-and accordingly the

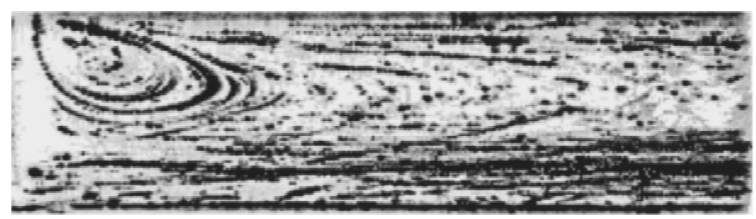

FIG. 6. This picture depicts the tracks of aluminum particles seeded in the fluid. For a small temperature gradient, such in this case, only the first roll, near the hot wall, is present. The photograph covers a zone of $8 \mathrm{~mm}$ high and $32 \mathrm{~mm}$ long (approximately one half of the cell). It should be noted that the shape of the roll changes with depth.

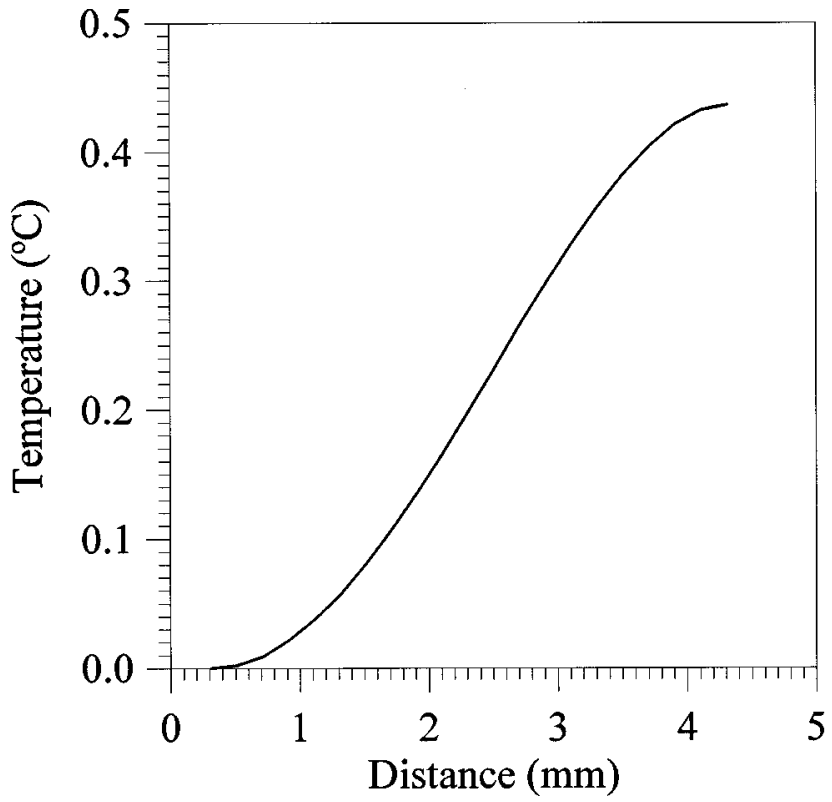

FIG. 7. Temperature profile of the wave shown in Fig. 5. It has been calculated following the same steps as in Fig. 2, taking a line that goes through approximately the middle of the wave.

waves-oscillate at about $1 \mathrm{~Hz}$. We propose an underlying mechanism that leads to the observed characteristic time of the instability, namely, the formation of a vertical thermal boundary layer. The time that it takes for a thermal boundary layer to develop along a vertical hot wall can be obtained from quite general assumptions [16]. If a sudden temperature step is applied to the wall, the characteristic growth time of

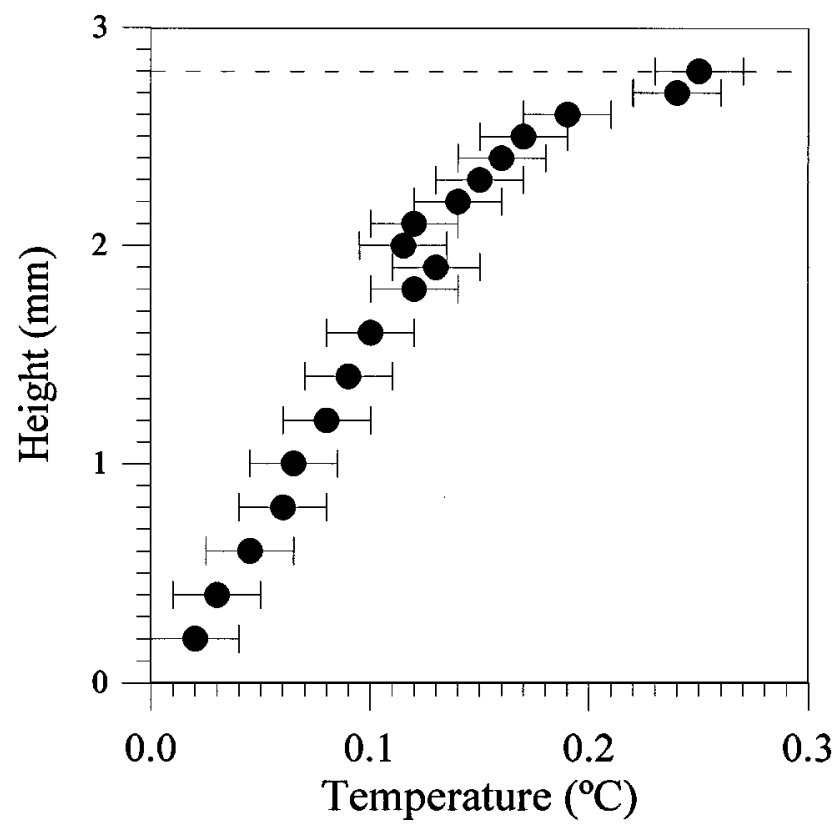

FIG. 8. The amplitude of the waves as a function of the depth. It has been obtained for a layer $2.8 \mathrm{~mm}$ high. The error bar is the standard deviation. In dynamic measurements, thermocouples introduce smaller deviations in the amplitude than in absolute measurements such as that of Fig. 3. It is clearly seen that the amplitude is larger near the surface. 


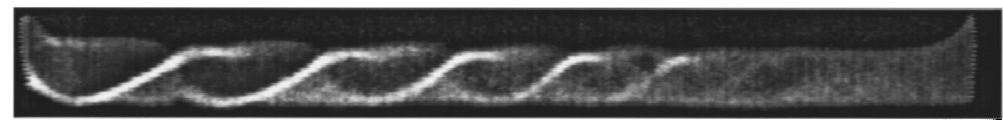

a)

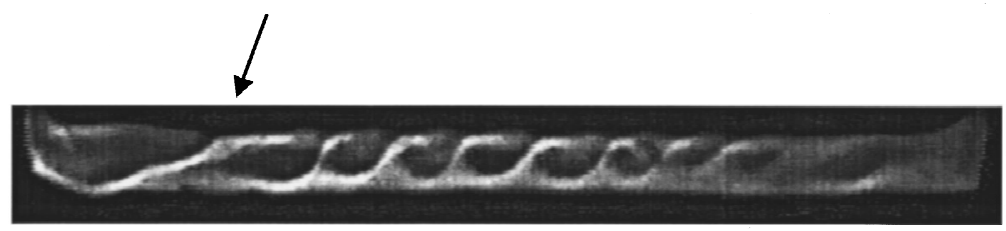

b)
FIG. 9. Shadowgraph pictures of the cell, taken from one side. The liquid layer is $3.1 \mathrm{~mm}$ high and $70 \mathrm{~mm}$ long. The hot side is at the left. In the upper picture, stationary rolls are seen. Their amplitude decreases as the distance from the hot wall increases. In the picture at the bottom, the temperature gradient is larger, and the first roll begins to oscillate. Apart from the first roll, all the other structures that can be seen in the shadowgraph are moving to the right, i.e., they are waves traveling toward the cold side. An arrow marks the position where waves detach from the first roll by the mechanism shown in Fig. 4. the boundary layer is found to be [17]

$$
\tau=\frac{h^{2}}{\kappa \mathrm{Ra}^{1 / 2}} .
$$

Schöpf and Patterson carried out an experiment to study the transient regime in a side-heated cavity, and found that the characteristic time for the development and destabilization of the vertical boundary layer agrees with this formula. In our experiment, the situation is different: the vertical wall is kept at a constant temperature, and the surface is free. But we suspect nevertheless that the mechanism might be quite similar. Formula (3) gives $1 \mathrm{~s}$ for our parameters, which is in agreement with the measured frequencies. But we are not able to provide further evidence in support of it. In the formula, $\tau$ does not depend on $h$, and in the experiments the dependence of $\tau$ on $h$ is very weak indeed within the limits of the experimental error [5]. In Eq. (3), $\tau$ depends on $\Delta T$ and the square root of the viscosity, but only small changes are accessible in the experiments. It should be remarked, however, that the tendency of $\tau$ is in agreement with formula (3) when the viscosity or the temperature difference are slightly varied [5].

The oscillations of the thermal boundary layer along a vertical hot plate is a well-known mechanism [18], and waves have been found in experiments carried out in closed cavities heated from the side $[19,20]$. We venture there are two processes that give rise to waves in a lateral container heated from the side: the hydrothermal instability, and the vertical boundary layer instability. This could explain the different propagation schemes observed in experiments.

\section{CONCLUSIONS}

We have produced evidence showing that there is a mechanism different from the hydrothermal instability giving rise to waves in a shallow liquid layer with a free surface heated from the side. The situation we presented can be viewed as a thermal boundary layer instability along the vertical hot wall. This instability can create a perturbation which is dragged downflow. This picture shares some features with the description provided by Bergé and Dubois of RayleighBénard convection [13], even if the experiments are not analogous. This alternative mechanism may explain the differences between the propagation schemes observed in some experiments that did not fit in theoretical descriptions.

\section{ACKNOWLEDGMENTS}

M. Dubois and H. Mancini suggested many of the ideas contained here. Helpful suggestions, aid, and comments from C. Pérez-García, J. Burguete, and D. Maza have improved this paper. P. Elizalde and B. Ozenda provided us technical help. Financial support from the European Union (Contract No. ERBCHRXT940546), from the Spanish Government (Contract No. PB95-0578), from the University of Navarre (PIUNA), and from Integrated French-Spanish Action Picasso No. 93024 is acknowledged.
[1] D. Villers and J. K. Platten, J. Fluid Mech. 234, 487 (1992).

[2] M. K. Smith and S. H. Davis, J. Fluid Mech. 132, 119 (1983); 132, 145 (1983).

[3] F. Daviaud and J. M. Vince, Phys. Rev. E 48, 4432 (1993).

[4] D. Schwabe, U. Möller, J. Schneider, and A. Scharmann, Phys. Fluids A 3, 2368 (1992).

[5] A. B. Ezersky, A. Garcimartín, J. Burguete, H. L. Mancini, and C. Pérez-García, Phys. Rev. E 47, 1126 (1993).

[6] C. De Saedeleer, A. Garcimartín, G. Chavepeyer, J. K. Platten, and G. Lebon, Phys. Fluids 8, 670 (1996).

[7] G. Z. Gershuni, P. Laure, C. M. Myznikov, B. Roux, and E. M. Zhukhovitsky, Microgravity Q 2, 142 (1992).
[8] P. Parmentier, V. Regnier, and G. Lebon, Int. J. Heat Mass Transf. 36, 2417 (1993).

[9] J.-F. Mercier and C. Normand, Phys. Fluids 8, 1433 (1996).

[10] A. B. Ezersky, A. Garcimartín, H. L. Mancini, and C. PérezGarcía, Phys. Rev. E 48, 4414 (1993).

[11] D. Malacara, Optical Shop Testing (Wiley, New York, 1979).

[12] P. Bergé and M. Dubois, in Scattering Techniques Applied to Supramolecular and Nonequilibrium Systems, edited by S.-H. Chen, B. Chu, and R. Nossal (Plenum, New York, 1981), p. 493.

[13] M. Dubois and P. Bergé, J. Phys. (France) 42, 167 (1981).

[14] J. Burguete, F. Daviaud, N. Mukolobwiez, and A. Garcimartín (unpublished). 
[15] When this article was in the process of refereeing, an article was published on the same subject: P. Gillon and G. M. Homsy, Phys. Fluids 8, 2953 (1996).

[16] L. Blumenfeld and E. Favre (private communication).

[17] J. Patterson and J. Imberger, J. Fluid Mech. 100, 65 (1980).
[18] H. Schlichting, Boundary-Layer Theory, 2nd ed. (McGrawHill, New York, 1987); H. Mancini and D. Maza, Phys. Rev. E 55, 2757 (1997).

[19] W. Schöpf and J. C. Patterson, J. Fluid Mech. 295, 357 (1995).

[20] R. J. A. Jansen and R. A. W. M. Henkes, Phys. Fluids 8, 62 (1996). 